\title{
A TEORIA DA COMPLEXIDADE E A FORMAÇÃO DE PROFESSORES DE LÍNGUAS ADICIONAIS
}

\author{
Paulo Ott TAVARES ${ }^{1}$ \\ Pontifícia Universidade Católica do Rio Grande do Sul \\ Cristina Becker LOPES-PERNA \\ Pontifícia Universidade Católica do Rio Grande do Sul ${ }^{2}$
}

RESUMO: O presente trabalho busca investigar em que medida os pressupostos da Teoria da Complexidade são discutidos durante a formação de futuros professores de línguas adicionais. A abordagem de fenômenos linguísticos a partir da perspectiva da complexidade foi introduzida na Linguística Aplicada por Larsen-Freeman (1997, 2002, 2008). No Brasil, Paiva (2006), Paiva e Borges (2011), Franco (2013), entre outros, têm estudado o ensino de línguas pelo viés da complexidade, e parece haver um número crescente de pesquisadores brasileiros que adotam tal perspectiva, resultando em trabalhos de mestrado e doutorado nesse campo. Uma questão que se coloca a partir da presença desse paradigma emergente na pesquisa em Linguística Aplicada no Brasil, é se essa produção está chegando na graduação, mais especificamente nos cursos de Letras, que formam professores de língua materna e adicional. Para discutir essa questão, o trabalho proposto faz uma revisão teórica das possibilidades de aplicação da complexidade no ensino de línguas adicionais, salientando conceitos próprios dessa abordagem que podem contribuir para o desenvolvimento da área. Em seguida é apresentada a metodologia da pesquisa realizada, a saber, questionários online respondidos por alunos de cursos de Letras em duas instituições de ensino superior em Porto Alegre/RS, que estão estudando para se tornarem professores de línguas adicionais. O questionário aplicado tem o objetivo de investigar o que os alunos sabem sobre o ensino de línguas adicionais através de uma abordagem complexa, e, em alguns casos, em qual momento de sua formação acadêmica eles foram apresentados a essa perspectiva. Resultados apontam que as contribuições da Teoria da Complexidade para a Linguística Aplicada ainda não são de conhecimento de grande parte dos alunos, o que mostra que ainda há um caminho a ser percorrido no sentido de popularizar a produção que já existe nos programas de pósgraduação no Brasil.

PALAVRAS-CHAVE: Ensino de língua adicional; formação de professores; teoria da complexidade.

ABSTRACT: This study aims to investigate to what extent Complexity Theory is discussed during the education of future teachers of additional languages. The approach to linguistic phenomena through a complexity perspective was first introduced in Applied Linguistics by Larsen-Freeman (1997, 2002, 2008). In Brazil, Paiva (2006), Paiva e Borges (2011), Franco (2013), among others, have adopted a complexity-informed approach to research language teaching and learning, and there seems to be a growing number of Brazilian researchers following that trend, which results in a series of master's theses and doctor's dissertations. However, it is yet to be known if that academic production is reaching undergraduate students, particularly those studying to become teachers of languages. In order to address that question, this study first defines what complex systems are, as well as the possible contributions of Complexity Theory to applied linguistics. Then, the research methodology is presented, namely, online questionnaires applied to undergraduate students training to become teachers of languages, in two universities in Porto Alegre/RS. The goal of the questionnaire was to explore what students know about language teaching through a complexity perspective, and when, during their university education, they were introduced to that approach. Results suggest that the contributions of Complexity Theory to applied linguistics

\footnotetext{
${ }^{1}$ Doutorando em Letras (Linguística) na PUCRS, com bolsa do CNPq.

${ }^{2}$ Professora-adjunta da Faculdade de Letras e do Programa de Pós-Graduação em Letras da PUCRS.
} 
are largely unknown to the students who took part in this study, showing that there is still a long way to go before the academic discussion and production found in graduate circles becomes familiar to undergraduate students.

KEYWORDS: Language teaching; teacher education; complexity theory.

\section{Introdução}

O paradigma da complexidade tem sido adotado cada vez mais por pesquisadores, tanto na Linguística Aplicada quanto em outras áreas do conhecimento, no Brasil e em outros países, nas investigações de seus fenômenos de interesse. Kramsch (2012, p.10), ao abordar o impacto da complexidade nas ciências sociais, chega a perguntar o porquê de estarem "todos tão empolgados com a teoria da complexidade na Linguística Aplicada"”. No cenário brasileiro, Franco (2013, p.184) afirma que "um número crescente de pesquisadores brasileiros [...] adota a complexidade como alternativa para investigar fenômenos complexos na Linguística Aplicada", e apresenta um resumo de pesquisas em nível de mestrado e doutorado realizadas nessa perspectiva nos últimos anos.

A partir da constatação desse crescimento de trabalhos e pesquisas que adotam a complexidade como forma de compreender fenômenos da linguagem, este trabalho busca discutir se essa perspectiva, já presente na produção em nível de pós-graduação, está chegando na graduação, especialmente nos cursos de Letras, que formam futuros professores de línguas (materna e adicional ${ }^{4}$ ). Para isso, um questionário foi aplicado com alunos de cursos de Letras de duas institutições de ensino superior no sul do Brasil, com o objetivo de investigar o quão familiarizados estes alunos estão com alguns conceitos e propostas da teoria da complexidade em relação à linguagem e ao ensino de línguas.

Inicialmente, será feita uma apresentação de definições e nomenclaturas importantes para a compreensão dessa proposta de investigação de fenômenos complexos, seguida de uma explicação sobre o que caracterizam os sistemas complexos. Em seguida, há uma aproximação entre a ciência da complexidade e fenômenos de ineteresse na área da Linguística Aplicada, especialmente aqueles relativos aos ensino e aprendizagem de línguas adicionais. A pesquisa realizada com alunos de cursos de Letras é então apresentada, e as respostas obtidas através do questionário são discutidas. Ao final do texto, são expressas as considerações finais, retomando os ponto discutidos anteriormente e apontando possibilidades futuras de pesquisa e discussão.

\section{Teoria da complexidade, sistemas complexos, abordagem complexa: algumas definições}

O presente trabalho faz uso de diferentes expressões para se referir a uma mesma postura frente aos fenômenos de interesse de pesquisa: teoria da complexidade, abordagem complexa, perspectiva da complexidade, complexidade, etc.. Mercer (2013, p.376) também explica essa variedade de termos semelhantes, e explica que

Sob o guarda-chuva das teorias da complexidade, há uma série de abordagens relacionadas, como teoria de sistemas, teoria do caos, sistemas adaptativos/dinâmicos complexos, sistemas emergentes, teoria de redes, dinâmica não-linear, entre outros. Entretanto, apesar das diferenças, todas

\footnotetext{
${ }^{3}$ A tradução dessa e das outras citações presnetes neste trabalho são de responsabilidades dos autores.

4 O termo "língua adicional" é adotado neste trabalho a partir da perspectiva discutida por Schlatter e Garcez (2009, 2012) e encontrada no documento da UNESCO intitulado Teaching Additional Languages (http://www.ibe.unesco.org/publications/EducationalPracticesSeriesPdf/prac06e.pdf).
} 
essas abordagens compartilham certas características fundamentais. Essencialmente, perspectivas complexas descrevem modelos orgânicos e holísticos, compostos de múltiplos sistemas. (grifo nosso)

Larsen-Freeman e Cameron (2008, p.1) destacam outro ponto, que deriva da colocação acima, e merece ser salientado: segundo as autoras, nessa perspectiva, "complexo" não significa apenas "complicado". A característica que define um sistema complexo é que o seu comportamento emerge da interação entre os seus componentes.

Portanto, a ideia de interação entre diferentes componentes/agentes é central em uma abordagem complexa de qualquer fenômeno de investigação, e esse princípio definidor vai permear as características dos sistemas complexos, que serão apresentadas a seguir. Antes disso, é importante traçar um breve histórico da disciplina da complexidade, a fim de contextualizar sua disseminação nos estudos da linguagem.

\section{Complexidade: uma nova forma de fazer ciência?}

A perspectiva complexa pode ser considerada como uma forma de romper com as formas mais tradicionais e clássicas de fazer ciência. Classicamente, a ciência tem adotado uma postura reducionista, buscando isolar e reduzir os objetos de estudo a fim de descrevê-los e explicá-los. A complexidade, ao assumir que a interação entre os diferentes componentes de um fenômeno é responsável por seu comportamento, inevitavelmente leva a uma nova forma de olhar e de encarar a investigação científica.

Martins e Braga (2007) apresentam um breve histórico e contextualização da perspectiva da complexidade na história do pensamento centífico, com destaque para a influência de René Descartes e Issac Newton no estabelecimento de um fazer científico. Segundo os autores, Descartes, ao estabelecer o início de uma sistematização na pesquisa científica, serviu de fundamentação para os estudos de Newton, e nesse momento a ciência clássica chega a seu ápice, marcando o começo de uma visão científico-filosófica do mundo. Na base dessa visão, está "a tentativa de se descrever o todo reduzindo-o à investigação das partes isoladas, dando origem ao que se denomina reducionismo" (MARTINS; BRAGA, 2007, p.218).

É com os estudos de física quântica, que despontam no final do século XIX, que começam a surgir novas correntes científico filosóficas, e é nesse contexto que surge a ciência da complexidade, que "inova ao propor uma visão holística que incorpora a não-linearidade, a imprevisibilidade, o dinamismo da relação entre as partes, a alta sensibilidade às condições iniciais e auto-organização de um fenômeno" (MARTINS; BRAGA, 2007, p.219).

As colocações acima já adiantam algumas das características dos sistemas complexos, que são abordados na próxima seção.

\section{O que são sistemas complexos?}

Para poder definir o que são sistemas complexos, é necessário, primeiramente, definir o que são sistemas (em geral), bem como o que diferencia um sistema complexo de um sistema simples. De acordo com Larsen-Freeman e Cameron (2008, p.25),

em uma definição abstrata e geral, um sistema é produzido por um conjunto de componentes que interagem de determinadas maneiras e produzem algum estado ou forma global em um momento particular. Sistemas se diferenciam 
de conjuntos, agregados, e coleções, na medida em que estar no sistema afeta as propriedades dos componentes (grifo nosso).

As mesmas autoras definem sistemas simples como "um pequeno grupo de componentes similares que estão conectados de formas previsíveis e que não se alteram" (LARSEN-FREEMAN; CAMERON, 2008, p.27). De maneira oposta, "sistemas complexos são formados por diferentes tipos de elementos, geralmente em grande quantidade, que estão conectados e interagindo de maneiras diferentes e que se alteram" (LARSEN-FREEMAN; CAMERON, 2008, p.26).

Alguns exemplos concretos e cotidianos que ilustram essas definições:

- uma coleção de livros: não é considerada um sistema, pois não há interação entre os componentes da coleção, e estar nesse grupo não afeta os elementos individualmente;

- um semáforo: é um sistema simples, pois os elementos que formam o sistema são semelhantes, e interagem de maneira previsível;

- um relógio: pode ser considerado um sistema simples (apesar de seu funcionamento ser, ou parecer, complicado para leigos), pois os elementos que formam suas engrenagens e comandam seu funcionamento interagem de forma programada, previsível, e sem alterações; - o trânsito de uma cidade/bairro: é um exemplo de sistema complexo, pois há uma grande quantidade de elementos de diferentes tipos (motoristas, pedestres, o clima, os carros, etc.), que interagem de maneira imprevisível. Esse exemplo mostra também, de forma clara, como estar em um sistema afeta o comportamento individual dos elementos que o compõem.

Uma característica importante dos sistemas complexos é que seus elementos podem ser, também, sistemas complexos. Larsen-Freeman e Cameron (2008, p.28) afirma que

se considerarmos uma comunidade de falantes um sistema complexo, haverá dentro dela grupos socioculturais que também funcionam como sistemas complexos; os indivíduos dentro desses sub-grupos podem ser vistos como sistemas complexos, assim como seus sistemas neurológicos. Há sistemas complexos em todo o percurso, do social ao neurológico.

A partir dessas definições, e da constatação de que a noção de interação é central em uma perspectiva complexa de abordagem de qualquer fenômeno, é possível discutir agumas características dos sistemas complexos, que serão importantes para sustentar a aproximação dessa abordagem aos estudos da linguagem e, principalmente, ao ensino e aprendizagem de línguas. Para isso, nos apoiamos nos estudos de Larsen-Freeman (1997, 2012, 2015), LarsenFreeman e Cameron (2008), e Van Lier (2004). De acordo com esse autores, sistemas complexos são:

- heterogêneos: os elementos/componentes/agentes de um sistema complexo são de diferentes tipos;

- dinâmicos: em um sistema complexo, as mudanças são constantes, e um estado futuro depende de alguma forma do estado presente;

- não-lineares: as relações de causa e efeito, em sistemas complexos, não são lineares, ou seja, os efeitos de determinada ação ou estado não são necessariamente proporcionais a suas causas; uma pequena alteração em algum parâmetro pode ter grandes consequências. Esta característica dos sistemas complexos também é cnhecida como o "efeito borboleta";

- abertos: sistemas complexos interagem com seu ambiente, podendo haver trocas de informação, energia, ou matéria, entre eles;

- adaptativos: sistemas adaptativos mudam em respostas a mudanças no ambiente;

- emergentes: um ordem complexa emerge, de maneira espontânea e imprevisível, da interação de seus componentes; 
- auto-organizados: a ordem que emerge não segue fatores externos, e não está prédeterminada em algum componente individual do sistema.

A partir dos conceitos de heterogeneidade, dinamismo, não-linearidade, abertura, adaptação, emergência, e auto-organização, pode-se chegar a duas constatações importantes e que têm papel de destaque ao trabalhar fenômenos da linguagem a partir de uma abordagem complexa: a) o todo é mais do que a soma das partes, o que leva a uma visão mais holística e que descarta o estudo de fenômenos isolados de seus contextos e circunstâncias; b) o contexto não é apenas o ambiente em torno de determinado fenômeno, mas parte integral do sistema, pois está em constante interação com tudo que acontece.

Com as características dos sistemas complexos definidas, pode-se discutir sua relação com estudos da linguagem e do ensino de línguas.

\section{Complexidade e ensino de línguas}

A apresentação da teoria da complexidade, e as características dos sistemas complexos expostas acima parecem mostrar uma relação natural entre essa perspectiva e a sala de aula, especialmente a aula de línguas. Pessoas familiares com esse ambiente possivelmente apontam muitas semelhanças entre as características apresentadas e sua experiência pessoal. Segundo Mercer (2013, p. 376),

parece que agora os pesquisadores estão explicitamente alinhados com o que professores(as) sempre souberam a partir de suas experiências de sala de aula: que o ensino de línguas é uma atividade extremamente complexa que apresenta aspectos pessoais, individuais, variados, e muitas vezes difíceis de prever em seu desenvolvimento.

A autora defende que o conceito de emergência "é especialmente visível, já que o comportamento da aula ou do grupo como um todo é emergente e depende da interação única, e situada temporalmente, de todos os indivíduos e outros componentes juntos na sala de aula" (MERCER, 213, p.379).

Do ponto de vista da pesquisa, Kramsch $(2012$, p.10) acredita que a perspectiva da complexidade pode se configurar em um campo de pesquisa unificado, estudando a aquisição ${ }^{5}$ da linguagem "através de uma perspectiva cognitiva, social, cultural, histórica, e até estética".

A adoção de uma visão orientada pela complexidade implica, também, na adoção de termos que sejam coerentes com essa postura. Larsen-Freeman (2015), defende que um termo compatível com essa proposta seja "desenvolvimento", e não "aquisição de língua". Segundo a autora, aquisição implica em uma visão linear, a transmissão de uma commodity que alguém tem ou não tem, e que há um ponto final no processo. O termo desenvolvimento reconheceria que a aprendizagem não é linear, que é caracterizada por progressos e regressos, e apresenta grande variação. A própria autora afirma que não espera que o termo aquisição seja substituído por desenvolvimento, mas defende que as ideias suscitadas por cada expressão merecem ser debatidas, e faz um convite à reflexão do que fundamenta as práticas de sala de aula dos professores de línguas.

\footnotetext{
5 Apesar de o termo "aquisição" ser consagrado nos estudos da linguagem, os autores deste trabalho acreditam que os termos "desenvolvimento", "aprendizagem", e "emergência" sejam mais apropriados a partir de uma perspectiva complexa. No entanto, citações que adotam o termo "aquisição" serão mantidas como na escrita original. Esta postura se alinha com a defendida por Martins e Braga (2007).
} 
Uma outra questão envolvendo terminologia é o uso do termo input, consagrado na literatura, e propiciamento (affordance, em inglês). O termo input está conectado ao termo aquisição, como algo que vai de algum lugar para a mente de quem aprende, e pressupõe uma relação de linearidade e causalidade. O termo propiciamento tem origem na psicologia ecológica de Gibson (2015), e é definido por Van Lier (2004, p.4) como "a relação entre um organismo (nesse caso, o aprendiz) e o ambiente, que sinaliza uma oportunidade ou restrição para a ação. O ambiente inclui todos os propiciamentos físicos, sociais e simbólicos que fornecem bases para a atividade" (grifo nosso). Propiciamento, então, seria tudo o que cerca o aluno, incluindo o professor, sua fala, as tarefas, o material didático, os colegas, a sala de aula, etc. Tudo no ambiente pode ser um disparador para uma ação que leve à aprendizagem.

Além de questões de nomenclatura, há outros elementos substanciais que embasam um trabalho com o ensino de línguas informado pela complexidade. $\mathrm{O}$ caráter não-linear dos sistemas complexos é outro forte ponto de diálogo entre essa perspectiva e a sala de aula de língua adicional. A partir do momento em que alunos e professores têm a consciência de que a aprendizagem não é um mero acúmulo de conteúdo, habilidades, e/ou conhecimentos, é possível que a dinâmica da sala de aula se torne diferente. Apesar de materiais didáticos e a estrutura tradicional do ensino estar construída de uma forma linear e progressiva, é importante que as pessoas envolvidas no processo de ensino e aprendizagem estejam cientes dos movimentos de progresso e regresso presentes no processo, até que a emergência de padrões mais estabilizados ocorra.

Van Lier (2004, p.196) apresenta algumas considerações a respeito da complexidade no ensino e aprendizagem de línguas, que estão alinhadas com o que foi exposto neste trabalho. Segundo o autor:

- "ensinar" não leva necessariamente, ou automaticamente, a "aprender";

- há diversas causas e razões para a aprendizagem. Algumas são previsíveis, mas também há acidentes e coincidências;

- a aprendizagem pode acontecer em qualquer lugar, a qualquer hora: na aula, entre aulas, em casa, ou na sala de aula;

- as causas não explicam sistemas complexos; conhecer as causas de algo não é o mesmo que entendê-lo;

- qualquer pequena mudança pode ter grandes consequências, ou nenhuma. O mesmo é verdadeiro para grandes mudanças.

A partir desse quadro, que mostra uma potencial aplicabilidade da teoria da complexidade no processo de ensino e aprendizagem, segue que é interessante investigar se esses temas vêm sendo discutidos na formação de professores de línguas, nos curso de graduação em Letras. A próxima seção apresenta uma pesquisa realizada com esse objetivo, seguida de uma discussão dos resultados.

\section{A teoria da complexidade na formação de professores de línguas adicionais}

Como exposto na abertura deste trabalho, parece haver um crescente (apesar de ainda incipiente) interesse de pesquisadores na Linguística Aplicada brasileira em usar os conceitos da teoria da complexidade em pesquisas de ensino e aprendizagem de línguas. Franco (2013) reconhece esse estágio inicial, mas aponta três pesquisadores que, na última década, se destacaram em relação a orientações de dissertações e teses nessa área. São eles: Paiva (UFMG), Freire (PUCSP), e Vetromille-Castro (UFPEL). Juntos, esses pesquisadores orientaram 23 trabalhos nesse período. 
Com o intuito de investigar se há discussão sobre a ciência da complexidade em nível de graduação em Letras, a pesquisa aqui proposta buscou verificar o grau de familiaridade de estudantes de graduação em Letras com alguns dos termos discutidos até aqui, como emergência, propiciamentos, sistemas complexos, não-linearidade, entre outros. O instrumento de pesquisa foi um questionário online divulgado entre estudantes de Letras nos últimos semestres do curso, em duas instituições de ensino superior no Sul do Brasil. Antes de apresentar os resultados do questionário, é importante ressaltar que, durante a realização da pesquisa, houve dificuldade no acesso aos estudantes, o que resultou em um baixo número de respostas. Apesar disso, decidiu-se continuar com o trabalho, com o intuito de promover a discussão do assunto e levantar novas questões de pesquisa.

O questionário foi respondido por seis estudantes, e os gráficos a seguir apresentam as perguntas e suas respostas:

1. Durante a sua formação na graduação, você lembra de ser apresentado, em algum momento, à Teoria da Complexidade?

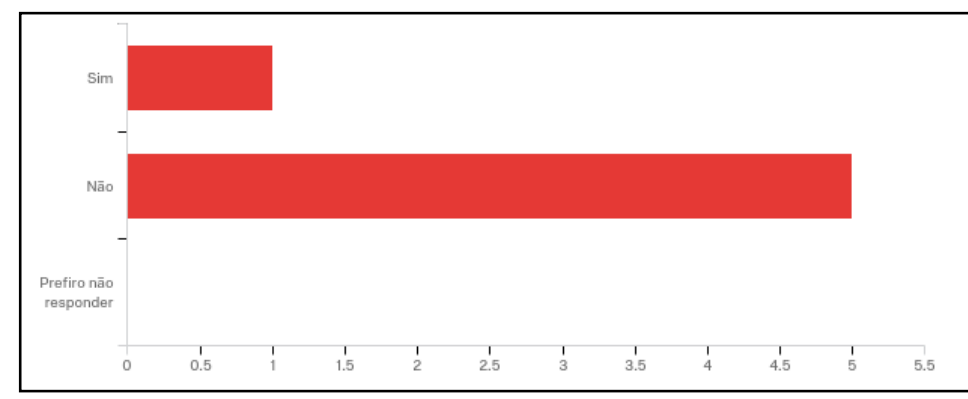

Gráfico 1: Conhecimento da Teoria da Complexidade

2. Durante as discussões sobre ensino e aprendizagem de línguas adicionais, você lembra de discutir o conceito de propiciamentos (affordances)?

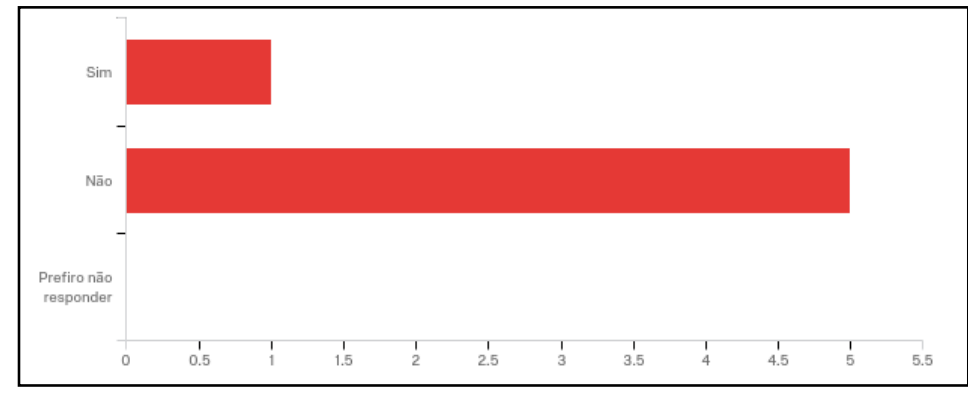

Gráfico 2: Conhecimento do conceito de propiciamento

3. Durante as discussões sobre ensino e aprendizagem de línguas adicionais, você lembra de discutir o conceito de input?

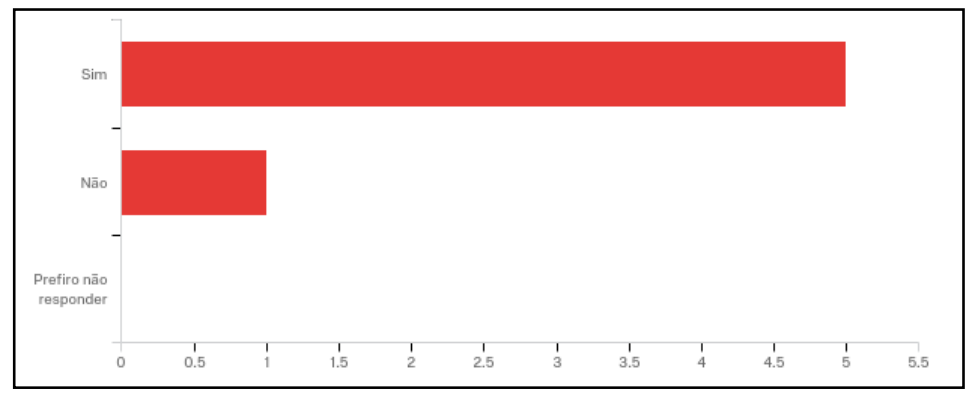

Gráfico 3: Conhecimento do conceito de input 
4. Qual dos seguintes termos você associa com mais naturalidade à discussão sobre ensino e aprendizagem de línguas adicionais?

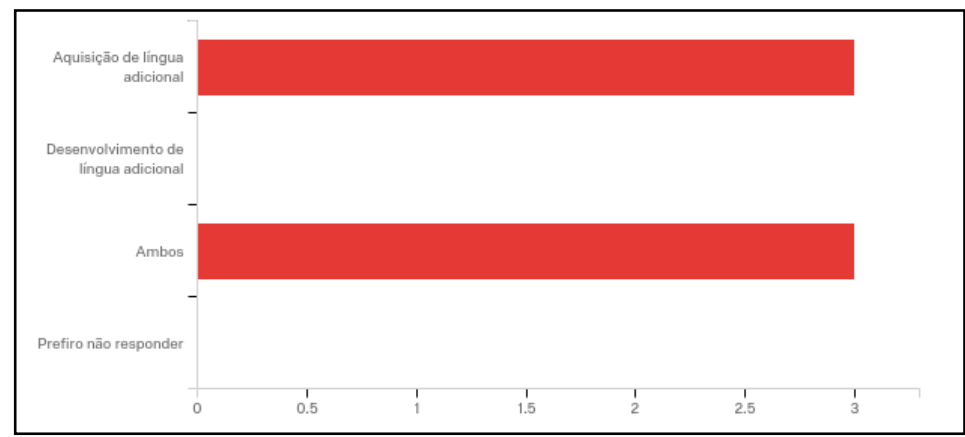

Gráfico 4: Aquisição ou desenvolvimento de língua adicional

5. Durante as discussões sobre ensino e aprendizagem de línguas adicionais, você lembra de discutir o conceito de emergência?

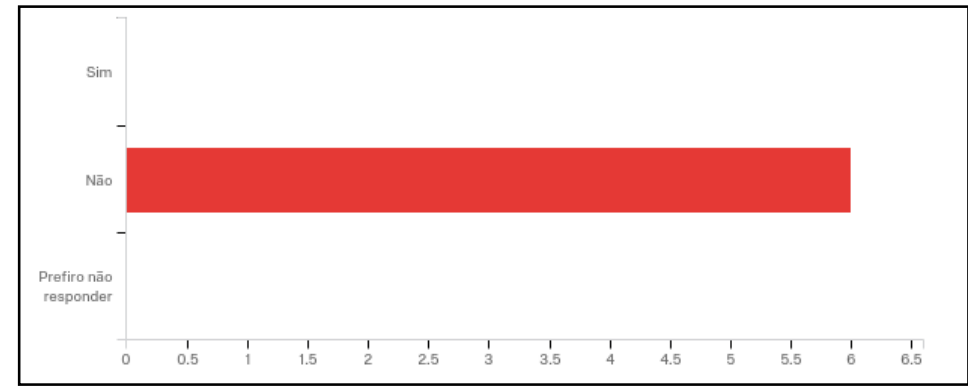

Gráfico 5: Conhecimento do conceito de emergência

6. Você concorda com a afirmação de que as línguas e o ensino de línguas são sistemas dinâmicos e complexos?

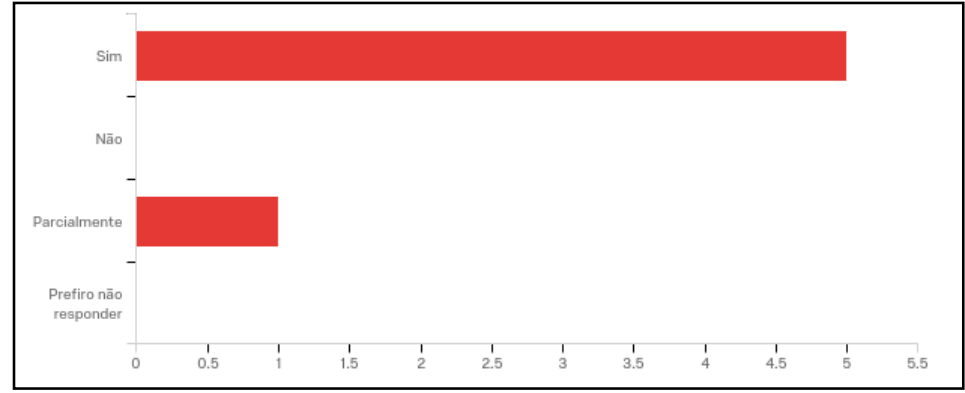

Gráfico 6: Língua como sistema complexo

7. Na sua visão, as línguas são sistemas abertos ou fechados?

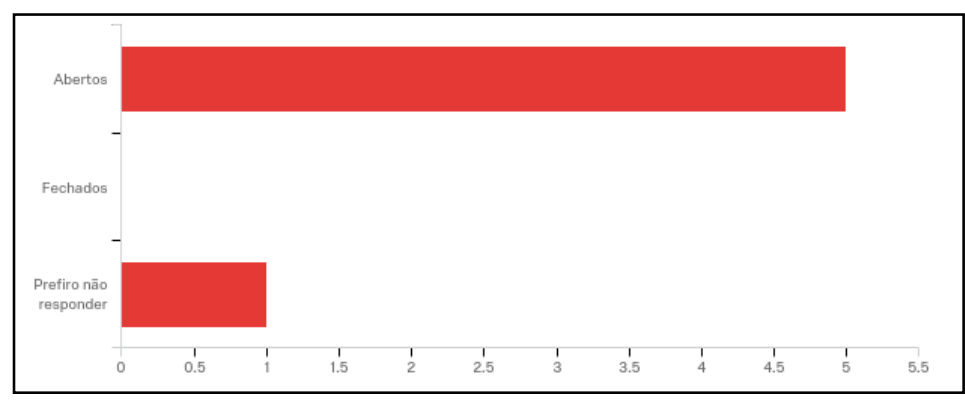

Gráfico 7: Língua como sistema aberto ou fechado 
8. Você considera que o processo de aprendizagem de uma língua é linear ou não-linear?

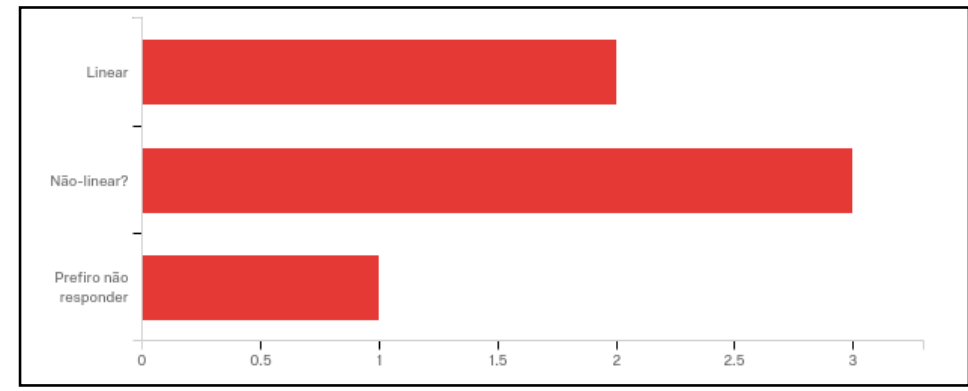

Gráfico 8: Aprendizagem linear ou não-linear

\section{Discussão das respostas}

Apesar do baixo número de respostas, é possível observar que a maioria dos estudantes não recorda ter sido apresentada, ou ter discutido, temas como a teoria da complexidade, propiciamentos, ou emergência. No entanto, quando perguntados sobre o termo input, estabelecido na área, as respostas mostram um padrão exatamente inverso, com a maioria reconhecendo ter estudado o conceito.

As outras questões buscaram investigar as percepções dos estudantes, que podem ter sido influenciadas pelas suas aulas na graduação, ou podem ser reflexo de seus sentimentos em relação a aspectos de ensino de línguas. Sobre os termos aquisição e desenvolvimento, observa-se que o termo aquisição é amplamente reconhecido, mas o termo desenvolvimento também é apontado. Mesmo sem discussão explícita de perspectivas complexas, é possível que os estudantes concordem com o termo desenvolvimento por considerarem que faz sentido ver a aprendizagem de línguas dessa forma.

Na mesma linha, as respostas mostram uma tendência a considerar as línguas sistemas dinâmicos e abertos, talvez refletindo, mais uma vez, experiências e crenças pessoais. Em relação à linearidade, as respostas são divididas, e não há como ver nenhum tipo de tendência.

Essas respostas, sempre salientando e reconhecendo que o baixo número de participantes compromete qualquer asserção mais definitiva sobre o tema, parecem sugerir que, apesar de não haver um trabalho explícito com os temas da complexidade nos cursos da grande maioria dos participantes, há uma tendência a concordar com alguns dos termos e expressões dessa perspectiva. É evidente que termos e nomenclaturas, por si só, não produzem consequências mais consistentes, mas seu reconhecimento por parte dos alunos pode indicar que, assim como afirma Tudor (2001, p.209), "poucos professores de línguas questionariam, de maneira séria, que o ensino de línguas é uma tarefa complexa". Esse pensamento, de fato, pode não estar acompanhado do embasamento que toda a reflexão teórica em torno da complexidade suscita, mas pode mostrar que há, nos alunos, a base para um trabalho nessa linha.

Outro ponto a destacar é que as respostas obtidas neste questionário refletem a experiência desses estudantes nas suas instituições de ensino. Sendo coerente com uma abordagem complexa dos fenômenos, não seria prudente afirmar que a realidade em outras instituições é exatamente a mesma. Seriam necessárias outras pesquisas para termos um quadro mais abrangente sobre a discussão da teoria da complexidade na formação de professores de línguas adicionais nos curso de Letras no Brasil. A experiência pessoal dos 
autores sugere que, de fato, ainda há um longo caminho até que essa perspectiva se torne mais popular, mas não há dados concretos para fazer essa afirmativa de forma mais categórica.

\section{Considerações finais}

O trabalho com o ensino e aprendizagem de línguas adicionais a partir de uma perspectiva complexa busca, como mencionado anteriormente, oferecer uma alternativa que aborde essa tarefa de uma maneira global, atentando para os diferentes fatores envolvidos. Tal iniciativa estaria alinhada com o que professores e demais envolvidos no processo de educação parecem pensar, até de maneira intuitiva, sobre a sala de aula de línguas.

Apesar do baixo número de respostas, a pesquisa sugere que estes participantes, mesmo não tendo estudado ou discutido as abordagens complexas de fenômenos linguísticos, parecem ter crenças que são coerentes com essa linha de pensamento. Da mesma maneira, Mercer (2013, p.394) diz que "muitos dos recentos avanços na linguística aplicada já um entendimento implícito da complexidade inerente dos processos de ensino e aprendizagem de línguas".

Além dos elementos técnico-científicos que a complexidade parece oferecer às explicações de fenômenos da linguagem, uma pedagogia que dialogue com essa visão de mundo permite que o "trabalho, inclusão, e valorização das diversidades individuais, das relações humanas, da sensibilidade contextual, e da qualidade de vida na sala de aula para todos os envolvidos" (MERCER, 2013, p.394).

Como conclusão deste trabalho, observa-se que há espaço e motivos para que a teoria da complexidade seja mais discutida nos cursos de graduação em Letras, para que os futuros professores de línguas adicionais tenham uma visão mais global, sensível, e compreensiva dos fenômenos de ensino e aprenidzagem. Krasmch (2012, p.21) resume bem o sentimento em relação às possibilidades e razões para um lugar mais destacada da complexidade na pesquisa e na formação de professores:

A teoria da complexidade desafia a ênfase excessiva colocada no desempenho competitivo e individual em nome da liberdade e da democracia. Também desafia a crescente pressão corporativa por uma padronização dos critérios de desempenho para uma maior rentabilidade econômica e para um maior controle da força de trabalho, em nome da produtividade e da eficiência. A teoria da complexidade também se coloca como uma ameça a uma comunidade científica em busca de credibilidade e legitimidade. Ao mesmo tempo em que melhora a validade das pesquisas em aquisição de língua, também torna as descobertas de tais pesquisas menos confiáveis e menos previsíveis, pois torna mais difícil isolar variáveis e estabelecer relações causais entre os fenômenos estudados. [...] A teoria da complexidade aplicada à educação linguística nos lembra dos complexos objetivos humanísticos do ensinoe aprendizagem de línguas.

\section{Referências}

FRANCO, C. de P. Um pouco de complexidade na Linguística Aplicada. Horizontes de linguística aplicada, ano 12, n.1, 2013, p. 183-197.

GIBSON, J. J. The ecological approach to visual perception. New York: Psychology Press, 2015. 
KRAMSCH, C. Why is everyone so excited about complexity theory in applied linguistics? In: BAILlY, S.; BOULTON, A.; MACARIE, D (Org.). Didactique des langues et comlexite. En hommage a Richard Duda. Melanges CRAPEL 33, 2012 p. 9-25.

LARSEN-FREEMAN, D. Chaos/complexity science and second language acquisition. Applied Linguistics. 18/2, 1997, p. 141-65.

LARSEN-FREEMAN, D. Complex, dynamic systems: A new transdisciplinary theme for applied linguistics?. Language Teaching, 45/2, 2012, p. 202-214.

LARSEN-FREEMAN, D. Saying what we mean: Making a case for 'language acquisition' to become 'language development'. Language Teaching, 48/4, 2015, p. 491-505.

LARSEN-FREEMAN, D.; CAMERON, L. Complex systems and applied linguistics. Oxford: Oxford University Press, 2008

MARTINS, A. C. S.; BRAGA, J. de C. F. Caos, complexidade e linguística aplicada: diálogos transdicsciplinares. Revista brasileira de linguística aplicada, v.7, n.2, 2001, p. 215-235.

MERCER, S. Towards a Complexity-Informed Pedagogy for Language Learning. Revista Brasileira de Linguística Aplicada. v.13, n.2, 2013, p. 375-398.

TUDOR, I. The Dynamics of the Language Classroom. Cambridge: Cambridge University Press, 2001.

van LIER, L. The ecology and semiotics of language learning: A sociocultural perspective. Boston: Kluwer Academic, 2004. 